\title{
Microcontroller Implementation, Chaos Control, Synchronization and Antisynchronization of Josephson Junction Model
}

\author{
Rolande Tsapla Fotsa ${ }^{a, 1^{*}}$, André Rodrigue Tchamda b,2, Alex Stephane Kemnang Tsafack c,3 \\ and Sifeu Takougang Kingni d,4 \\ ${ }^{a}$ Department of Mechanical Engineering, College of Technology, University of Buea, P. O. Box: 63 Buea, Cameroon \\ ${ }^{b}$ Department of Rural Engineering, Faculty of Agronomy and Agricultural Sciences, University of Dschang, P. O Box \\ 222, Dschang, Cameroon \\ ${ }^{\mathrm{c}}$ Research unit of Condensed Matter of Electronics and Signal Processing, Department of Physics, Faculty of Sciences, \\ University of Dschang, P. O. Box 67, Dschang, Cameroon \\ ${ }^{d}$ Department of Mechanical, Petroleum and Gas Engineering, Faculty of Mines and Petroleum Industries, University of \\ Maroua, P. O. Box 46, Maroua, Cameroon \\ ${ }^{1}$ rtsapla@yahoo.com; ${ }^{2}$ rodriguetchamda@yahoo.fr; ${ }^{3}$ alexstephanekemnang@gmail.com; ${ }^{4}$ stkingni@ gmail.com \\ * Corresponding author
}

\section{ARTICLE INFO}

Article history

Received 29 May 2021

Revised 29 June 2021

Accepted 08 July 2021

Keywords

Josephson Junction;

Hidden Chaotic Attractor;

Microcontroller

Implementation;

Chaos control;

Synchronization;

Antisynchronization

\section{ABSTRACT}

The microcontroller implementation, chaos control, synchronization, and antisynchronization of the nonlinear resistive-capacitiveinductive shunted Josephson junction (NRCISJJ) model are reported in this paper. The dynamical behavior of the NRCISJJ model is performed using phase portraits and time series. The numerical simulations result reveal that the NRCISJJ model exhibits different shapes of hidden chaotic attractors by varying the parameters. The existence of different shapes of hidden chaotic attractors is confirmed by microcontroller results obtained from the microcontroller implementation of the NRCISJJ model. It is theoretically demonstrated that the two designed single controllers can suppress the hidden chaotic attractors found in the NRCISJJ model. Finally, the synchronization and antisynchronization of unidirectional coupled NRCISJJ models are studied by using the feedback control method. Thanks to the Routh Hurwitz stability criterion, the controllers are designed in order to control chaos in JJ models and achieved synchronization and antisynchronization between coupled NRCISJJ models. Numerical simulations are shown to clarify and confirm control, synchronization, and antisynchronization.

This is an open-access article under the CC-BY-SA license.

\section{Introduction}

The discovery of new effects in superconductive tunneling in 1962, commonly known as Josephson effect in Josephson junctions (JJs) has attracted the attention of the scientific community due to the huge spectrum of applications in various domains of science and engineering. A JJ is made up of two superconductors sandwiched by a thin insulating layer 
where electrons can cross through the insulating layer [1]. The current (called a Josephson current) proportional to the phase difference between the two superconductors, which flow through the junction in the absence of an applied voltage and the movement of electrons across the insulating barrier, is known as Josephson tunneling [2, 3]. JJ is useful in constructing superconducting electronic devices such as terahertz pulse generator $[4,5]$, a millimeter or submillimeter wave oscillator [6], in digital systems [7], parametric amplifiers, voltage standards, in SQUID for detection of very weak magnetic fields $[5,8,9]$ and superconducting quantum interference devices such as magnetometer applications [10]. Many models have been used to study, characterize and represent the JJ properties. These include the nonlinear resistive-capacitive shunted JJ (NRCSJJ) model, the linear resistive-capacitive shunted JJ (LRCSJJ) model, linear resistive-capacitive-inductive shunted JJ (LRCISJJ) model [11-13], the nonlinear resistive-capacitive-inductive shunted JJ (NRCISJJ) model [14-20] autonomous Josephson jerk oscillator [15], and a single mesh JJ [16] just to name a few. It has been found that the NRCSJJ and LRCSJJ models show chaotic behaviors when they are driven by an external sinusoidal signal [14]. In comparison, the LRCISJJ and NRCISJJ models can generate chaotic behaviors with external DC bias [17-19]. The NRCISJJ and LRCISJJ models significantly reproduce the features on experimental curves than NRCSJJ and LRCSJJ models [17, 22, 23]. Many works showing multiples attractors phenomena have been carried out in the literature, in particular, Infinite attractors in a chaotic circuit with exponential memristor and JJ resonator [21]. In [18], it has been demonstrated that the LRCISJJ model can exhibit hidden chaotic attractors and the coexisting attractors between periodic and hidden chaotic attractors for a suitable choice of the parameters. In this paper, a microcontroller implementation is used to confirm the existence of different shapes of hidden chaotic attractors found in the NRCISJJ model during the numerical simulations.

However, in most applications of the JJ, such as in parametric amplifiers, voltage standard, and SQUID is essential to avoid all types of noise and chaos. Hence there is a need to control chaos in JJs. Chaos control in JJ models has been implemented with several methods such as the backstepping control method [24-26], sliding mode control method [27], linear feedback control method [28], and adaptive feedback control method [29]. The controllers designed in [24-29] to suppress chaotic behavior in JJ models are nonlinear and complicated.

Motivated by the above works and focus on the importance of the NRCISJ model, we shed more light on the studies of simple, concise, and single-state feedback controllers to control chaos in JJ models via a single feedback controller. Moreover, synchronization and antisynchronization of NRCISJJ models are investigated.

The paper is articulated around five sections presented as follows: The description and microcontroller implementation of NRCISJJ are presented in Section 2. Section 3 focuses on Chaos control of the NRCISJJ model by using a single feedback controller. Section 4 deals with the synchronization and antisynchronization of unidirectional coupled NRCISJJ models. Finally, the conclusion of the paper is drawn in Section 5.

\section{Description and microcontroller implementation of NRCISJJ model}

The dimensionless rate of equations describing an NRCISJJ model is given by [21]:

$$
\begin{gathered}
\frac{d x}{d t}=\frac{1}{\beta_{C}}[i-y-g(x) x-\sin (z)], \\
\frac{d y}{d t}=\frac{1}{\beta_{L}}(x-y),
\end{gathered}
$$




$$
\frac{d z}{d t}=x
$$

Where $t, x, y, z$ represent the dimensionless time, the voltage in the junction, the inductive current, and the phase difference, respectively. The parameter $i$ is direct external current and $\beta_{C}, \beta_{L}$ are capacitive and inductive parameters, respectively. The function $g(x)$ is a piecewise function approximation by a current-voltage characteristic of the intrinsic junction shunt resistor and is defined by:

$$
g(x)=\left\{\begin{array}{ll}
0.366 & \text { if }|x|>2.9 \\
0.061 & \text { if }|x| \leq 2.9
\end{array} .\right.
$$

The NRCISJJ model has two equilibrium points $E_{1}(0,0, \arcsin (i))$, and $E_{2}(0,0, \pi-\arcsin (i))$ for $i \leq 1$ a while $i>1$, it has no equilibrium points. For $i \leq 1$, the NRCISJJ model belongs to the family of systems with self-excited attractors, whereas for $i>1$, it belongs to the family of systems with hidden attractors. For $\beta_{C}=0.707$, it is depicted in Fig. 1 the time evolutions and the phase planes of hidden chaotic attractors for given values of parameters $i$ and $\beta_{L}$.

(a)

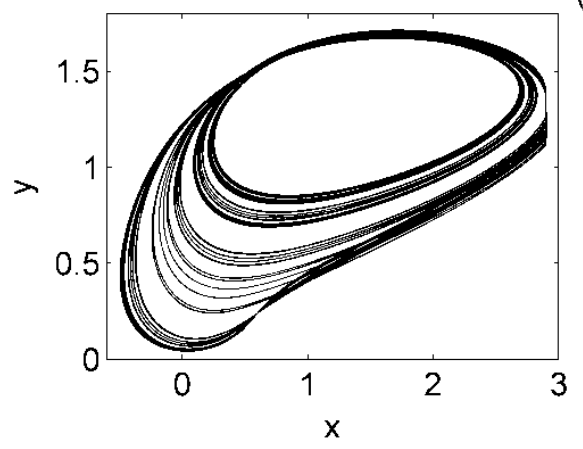

(c)

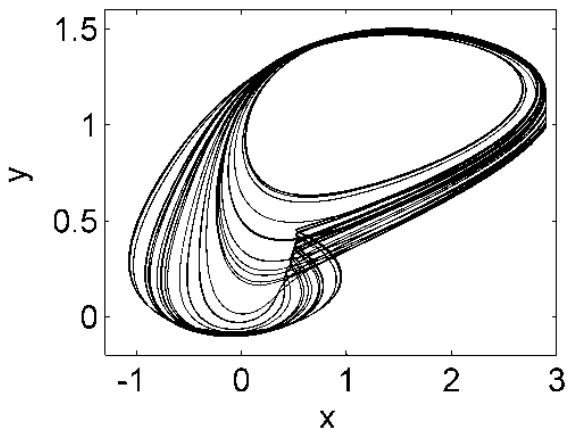

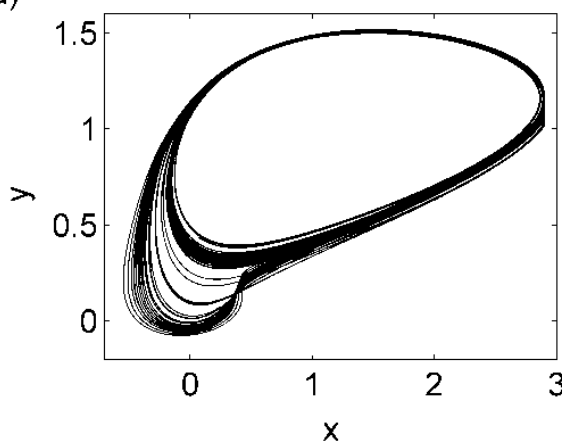

(b)

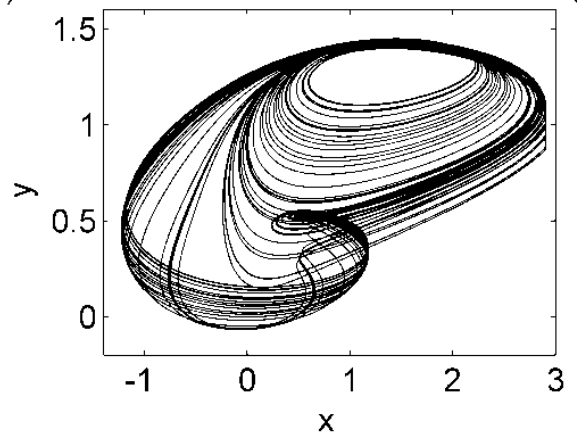

(d)
Fig. 1. Phase planes of NRCISJJ model for given values of parameters $i$ and $\beta_{L}$ :(a) $i=1.36$,

$\beta_{L}=2.07$, (b) $i=1.15, \beta_{L}=2.52$, (c) $i=1.15, \beta_{L}=2.6$ and (d) $i=1.15, \beta_{L}=3$. The other parameter is $\beta_{C}=0.707$. The initial conditions are $(\mathrm{x}(0), \mathrm{y}(0), \mathrm{z}(0))=(0,0,0)$.

Fig. 1 shows four different shapes of hidden chaotic attractors for given values of parameters $i$ and $\beta_{L}$. The microcontroller implementation of the NRCISJJ model is presented in Fig. 2. 


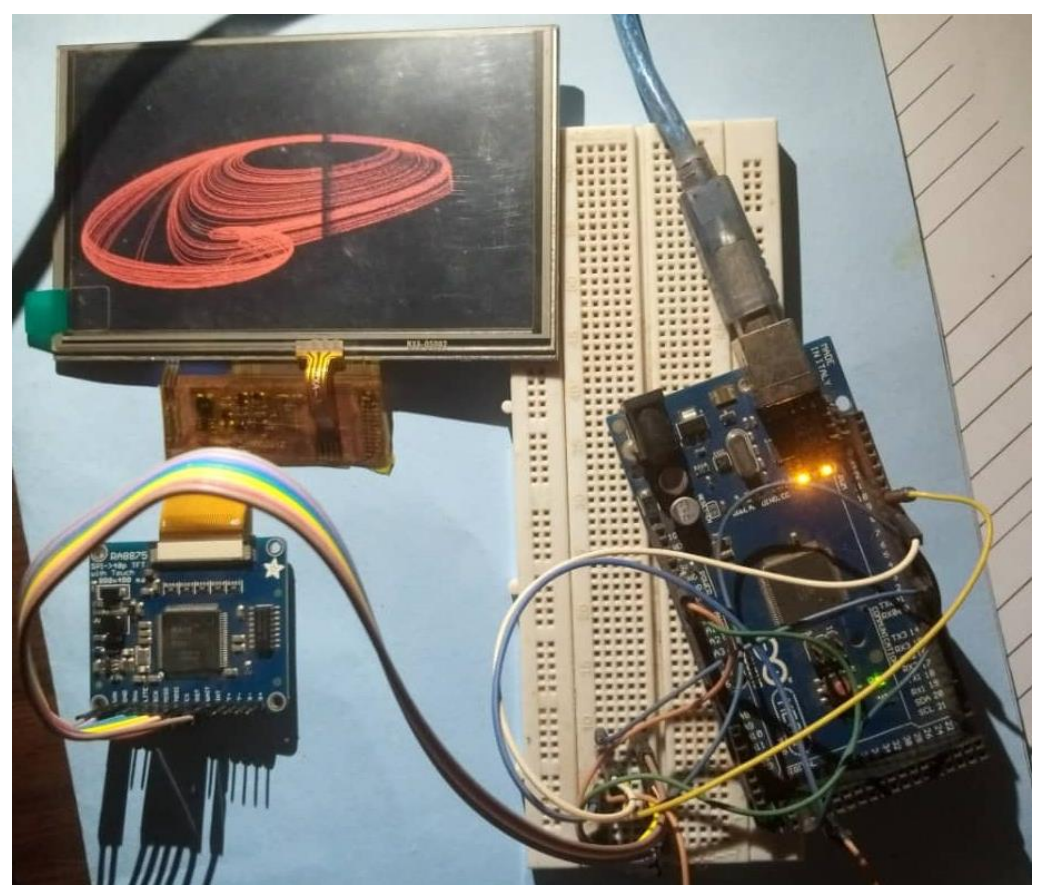

Fig. 2. Microcontroller design circuit of NRCISJJ model.

The microcontroller implementation of the NRCISJJ model shown in Fig. 2 is based on the Arduino mega 2560 card clocked at $16 \mathrm{MHz}$. NRCISJJ model described by the system (1) is sampled by the fourth-order Runge Kutta method. Fig. 3 presents the phase planes obtained from the microcontroller implementation of the NRCISJJ model.
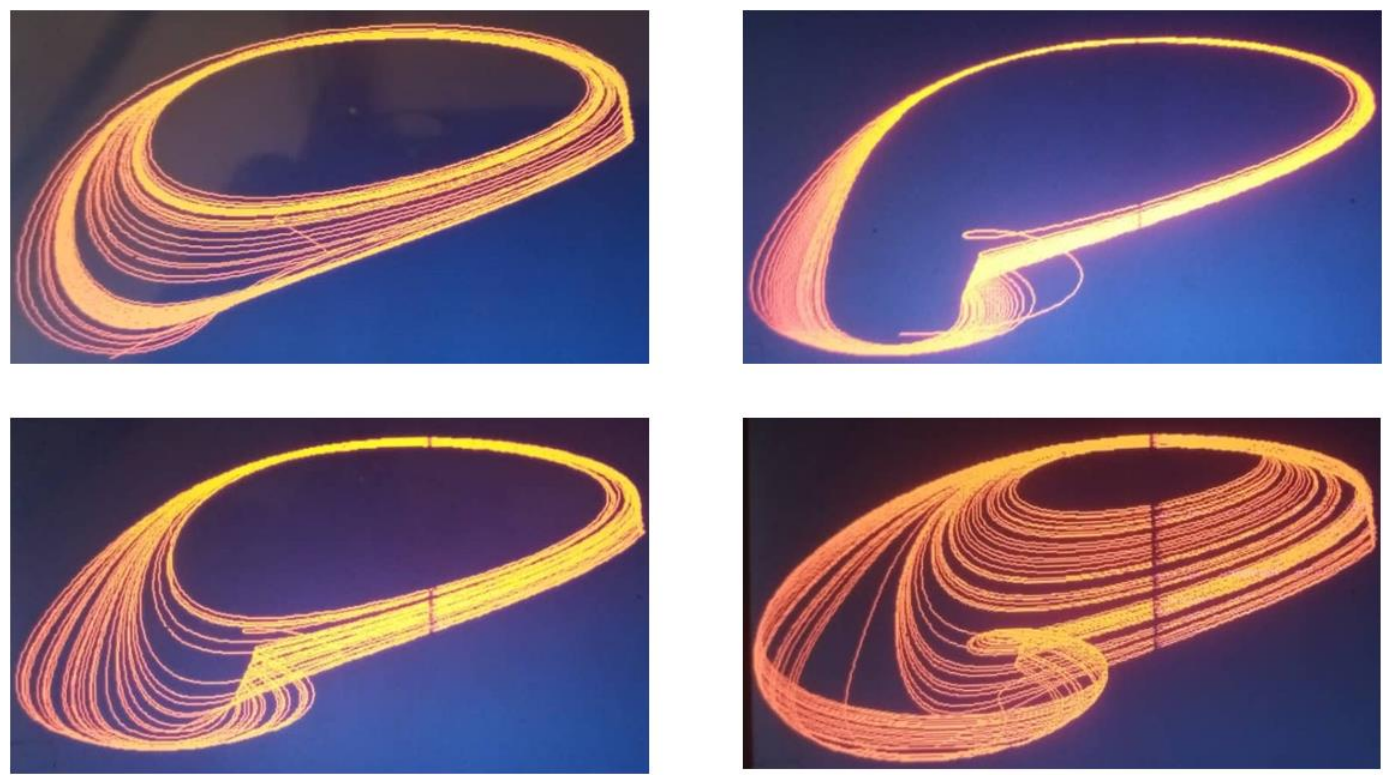

Fig. 3. Phase planes were obtained from the microcontroller implementation of the NRCISJJ model by using the same parameters as in Fig 1.

The microcontroller results of Fig. 3 are qualitatively agreed with the results of the numerical simulation of Fig. 1. Therefore, the existence of different shapes of hidden chaotic attractors found in the NRCISJJ model is confirmed. 


\section{Chaos Control of NRCISJJ model}

This section presents the control of hidden chaotic attractors found in the NRCISJJ model by designing two single controllers.

\subsection{First designed controller}

System (1) under the controller $u_{1}$ becomes:

$$
\begin{gathered}
\frac{d x}{d t}=\frac{1}{\beta_{C}}[i-y-g(x) x-\sin (z)]+u_{1}, \\
\frac{d y}{d t}=\frac{1}{\beta_{L}}(x-y), \\
\frac{d z}{d t}=x,
\end{gathered}
$$

whereby choosing $u_{2}=-x-[i-y-g(x) x-\sin (z)] / \beta_{C}$, system (3) becomes:

$$
\begin{gathered}
\frac{d x}{d t}=-x, \\
\frac{d y}{d t}=\frac{1}{\beta_{L}}(x-y), \\
\frac{d z}{d t}=x .
\end{gathered}
$$

The solution of (4a) is $x(t)=x(0) e^{-t}$. Thereafter $\lim _{t \rightarrow \infty} x(t)=0$ and system (4) is reduced to:

$$
\begin{gathered}
\frac{d y}{d t}=-\frac{1}{\beta_{L}} y, \\
\frac{d z}{d t}=0 .
\end{gathered}
$$

The solutions of (5a) and (5b) are $y(t)=y(0) e^{-t / \beta_{L}}$ and $z(t)=z(0)$, respectively. Thereafter $\lim _{t \rightarrow \infty} y(t)=0, \lim _{t \rightarrow \infty} z(t)=z(0)$ and the hidden chaotic attractor found in the NRCISJJ model can be controlled by using the controller, $u_{2}=-x-\left[i-y-\beta_{R} x-\sin (z)\right] / \beta_{C}$ as shown in Fig. 4 that demonstrates that the control of chaos in the NRCISJJ model by using the controller $u_{1}=-x-\left[i-y-\beta_{R} x-\sin (z)\right] / \beta_{C}$ is effective.

\subsection{Second designed controller}

System (1) under the controller $u_{2}$ becomes:

$$
\begin{gathered}
\frac{d x}{d t}=\frac{1}{\beta_{C}}[i-y-g(x) x-\sin (z)], \\
\frac{d y}{d t}=\frac{1}{\beta_{L}}(x-y), \\
\frac{d z}{d t}=x+u_{2},
\end{gathered}
$$


where by choosing $u_{2}=-x-z$, system (6) becomes:

$$
\begin{gathered}
\frac{d x}{d t}=\frac{1}{\beta_{C}}[i-y-g(x) x-\sin (z)], \\
\frac{d y}{d t}=\frac{1}{\beta_{L}}(x-y), \\
\frac{d z}{d t}=-z .
\end{gathered}
$$
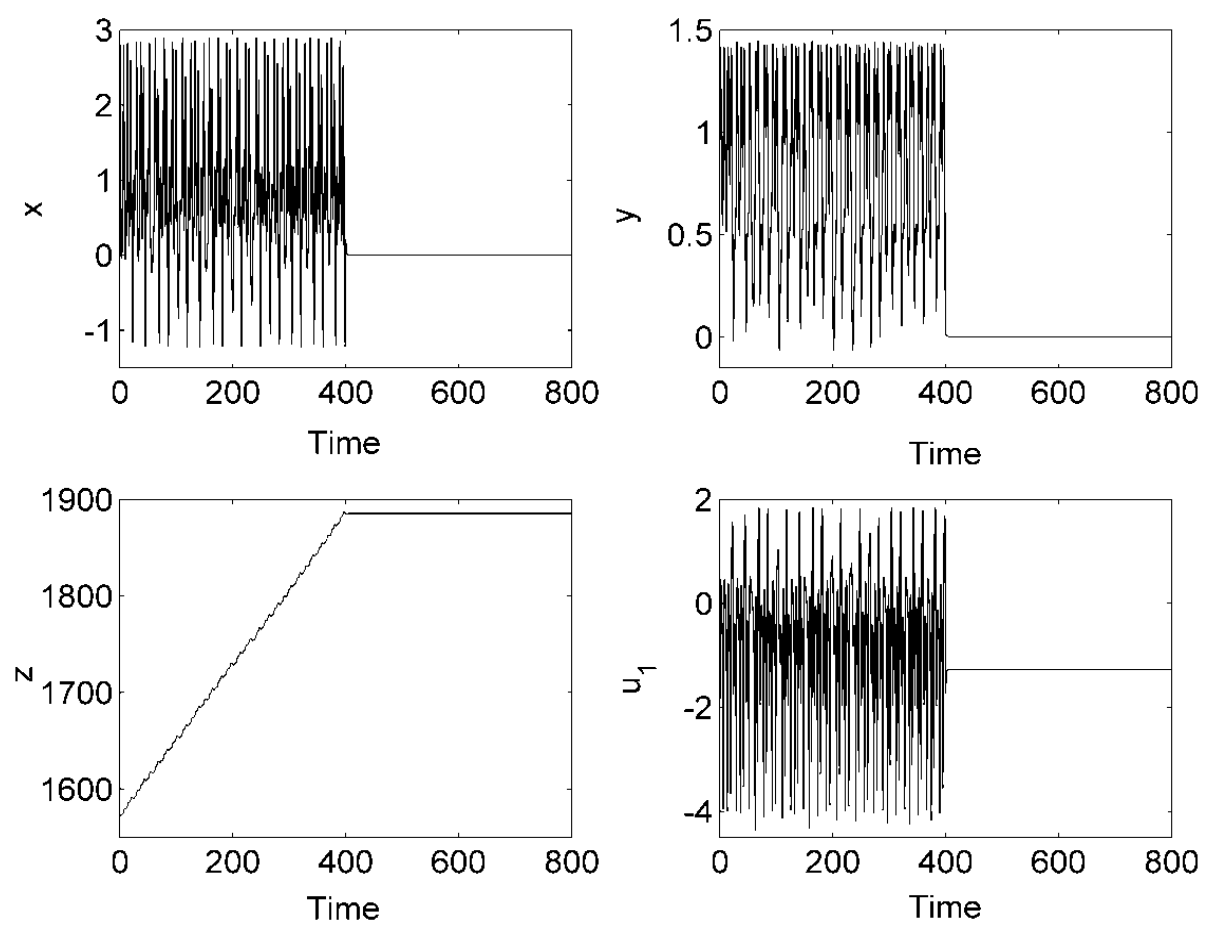

Fig. 4. Time evolutions of $x, y, z$ and $u_{1}$ showing the control scheme of the first controller for the parameters taking as $i=1.15, \beta_{C}=0.707, \beta_{L}=3$, the initial conditions are $(\mathrm{x}(0), \mathrm{y}(0), \mathrm{z}(0))=(0,0$, 0 ) and the controller $u_{1}$ is activated at $t \geq 400$.

The solution of (7c) is $z(t)=z(0) e^{-t}$. Thereafter $\lim _{t \rightarrow \infty} z(t)=0$ and system (7) is reduced to:

$$
\begin{gathered}
\frac{d x}{d t}=\frac{1}{\beta_{C}}[i-y-g(x) x], \\
\frac{d y}{d t}=\frac{1}{\beta_{L}}(x-y) .
\end{gathered}
$$

For $i=1.15, \beta_{C}=0.707, \beta_{L}=3$, the eigenvalues associated with the system (8) at the equilibrium point $\left(x=\frac{i}{1+0.366}, y=\frac{i}{1+0.366}\right)$ are $\lambda_{1,2}=-0.4255068364 \pm 0.680421637 j$ if $|x|>2.9$ with $j^{2}=-1$. While for $|x| \leq 2.9$, the eigenvalues associated to system (8) at the equilibrium point $\left(x=\frac{i}{1+0.061}, y=\frac{i}{1+0.061}\right)$ are $\lambda_{1,2}=-0.20980669 \pm 0.6754382937 j$. Since, all the real parts of the eigenvalues $\lambda_{1,2}$ are negative, the system (8) is asymptotically stable. 
Therefore, the hidden chaotic attractor found in NRCISJJ model can be controlled by using the controller, $u_{2}=-x-z$ as shown in Fig. 5 that demonstrates that the control of chaos in NRCISJJ model by using the controller $u_{2}=-x-z$ is effective.
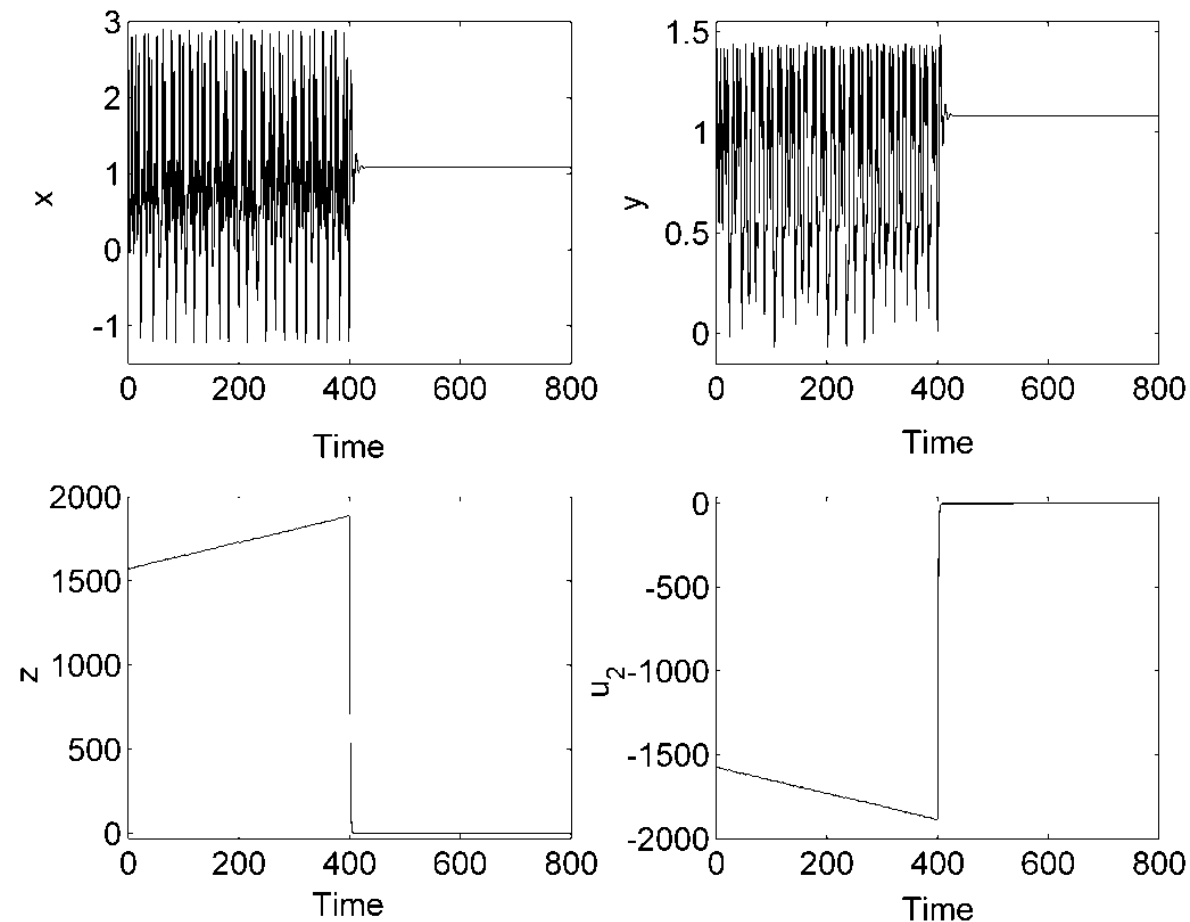

Fig. 5. Time evolutions of $x, y, z$ and $u_{2}$ showing the control scheme of the second controller for the parameters taking as $i=1.15, \beta_{C}=0.707, \beta_{L}=3$, initial conditions are $(\mathrm{x}(0), \mathrm{y}(0), \mathrm{z}(0))=(0,0,0)$ and The controller $u_{2}$ activated at $t \geq 400$.

\section{Synchronization and antisynchronization of coupled NRCISJJ models}

A feedback control method is used in this section to achieve synchronization and antisynchronization in unidirectional coupled identical NRCISJJ models. The drive and response NRCISJJ models are presented respectively:

$$
\begin{gathered}
\frac{d x_{1}}{d t}=\frac{1}{\beta_{C}}\left[i-y_{1}-g\left(x_{1}\right) x_{1}-\sin \left(z_{1}\right)\right], \\
\frac{d y_{1}}{d t}=\frac{1}{\beta_{L}}\left(x_{1}-y_{1}\right), \\
\frac{d z_{1}}{d t}=x_{1}, \\
\frac{d x_{2}}{d t}=\frac{1}{\beta_{C}}\left[i-y_{2}-g\left(x_{2}\right) x_{2}-\sin \left(z_{2}\right)\right]+u_{3}, \\
\frac{d y_{2}}{d t}=\frac{1}{\beta_{L}}\left(x_{2}-y_{2}\right), \\
\frac{d z_{2}}{d t}=x_{2}+u_{4},
\end{gathered}
$$


Where $u_{3}$ and $u_{4}$ are the controllers to be designed. The error variables are defined as $e_{1}=x_{2}-q x_{1}, e_{2}=y_{2}-q y_{1}, e_{3}=z_{2}-q z_{1}$ where $q=1$ for synchronization and $q=-1$ for antisynchronization. Its derivatives with respect to the time are given below:

$$
\begin{gathered}
\frac{d e_{1}}{d t}=\frac{1}{\beta_{C}}\left[i-y_{2}-g\left(x_{2}\right) x_{2}-\sin \left(z_{2}\right)\right]-\frac{q}{\beta_{C}}\left[i-y_{1}-g\left(x_{1}\right) x_{1}-\sin \left(z_{1}\right)\right]+u_{3}, \\
\frac{d e_{2}}{d t}=\frac{1}{\beta_{L}}\left(e_{1}-e_{2}\right), \\
\frac{d e_{3}}{d t}=e_{1}+u_{4},
\end{gathered}
$$

where the controllers are chosen as $u_{3}=-e_{1}-\left[i-y_{2}-g\left(x_{2}\right) x_{2}-\sin \left(z_{2}\right)\right] / \beta_{C}+q\left[i-y_{1}-g\left(x_{1}\right) x_{1}-\sin \left(z_{1}\right)\right] / \beta_{C}$ and $u_{4}=-e_{1}-e_{3}$ in order to achieve synchronization and antisynchronization between systems (9) and (10), by substituting the controllers $u_{3}$ and $u_{4}$ in the system (11), it becomes:

$$
\begin{gathered}
\frac{d e_{1}}{d t}=-e_{1}, \\
\frac{d e_{2}}{d t}=\frac{1}{\beta_{L}}\left(e_{1}-e_{2}\right), \\
\frac{d e_{3}}{d t}=-e_{3} .
\end{gathered}
$$

The solutions of (12a) and (12c) are $e_{1}(t)=e_{1}(0) e^{-t}$ and $e_{3}(t)=e_{3}(0) e^{-t}$. That is yield $\lim _{t \rightarrow \infty} e_{1}(t)=0, \lim _{t \rightarrow \infty} e_{3}(t)=0$ and system (12) can be reduced to:

$$
\frac{d e_{2}}{d t}=-\frac{1}{\beta_{L}} e_{2}
$$

The solution of (13) is $e_{2}(t)=e_{2}(0) e^{-t / \beta_{L}}$. That is yield $\lim _{t \rightarrow \infty} e_{2}(t)=0$ and the designed controllers $u_{3}=-e_{1}-\left[i-y_{2}-g\left(x_{2}\right) x_{2}-\sin \left(z_{2}\right)\right] / \beta_{C}+q\left[i-y_{1}-g\left(x_{1}\right) x_{1}-\sin \left(z_{1}\right)\right] / \beta_{C}$ and $u_{4}=-e_{1}-e_{3}$ can achieve synchronization or antisynchronization between the drive system (9) and the response system (10), as shown in Fig. 6 and Fig. 7. For numerical simulations, the initial conditions of the drive system (9) and response system (10) are $\left(x_{1}(0), y_{1}(0), z_{1}(0)\right)=(1,1,1)$ and $\left(x_{2}(0), y_{2}(0), z_{2}(0)\right)=(2,2,2), \quad$ respectively. $\quad$ By choosing $q=1$, the time evolutions of error variables and synchronization diagrams are presented in Fig. 6.

In Fig. 6, it is possible to achieve the synchronization between the drive system (9) and response system (10) by using the designed controllers $u_{3}$ and $u_{4}$. By choosing $q=-1$, the time evolutions of error variables and the outputs of the drive and response systems are presented in Fig. 7. It emerges from Fig. 7. It is possible to achieve the antisynchronization between the drive system (9) and response system (10) using the designed controllers $u_{3}$ and $u_{4}$. 
(a)
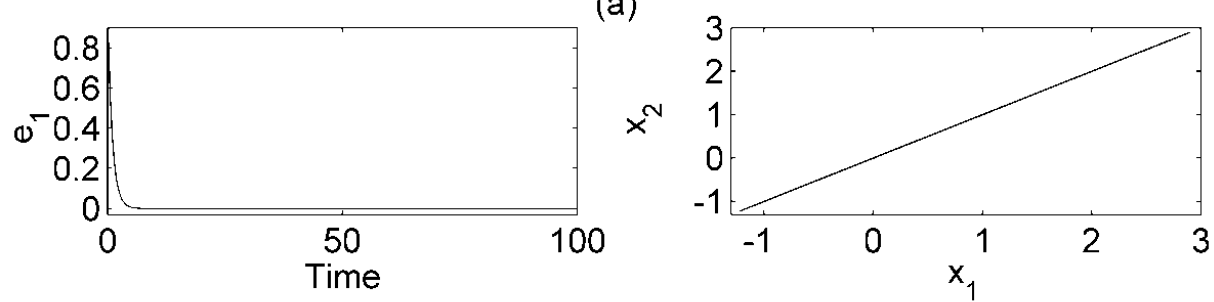

(b)
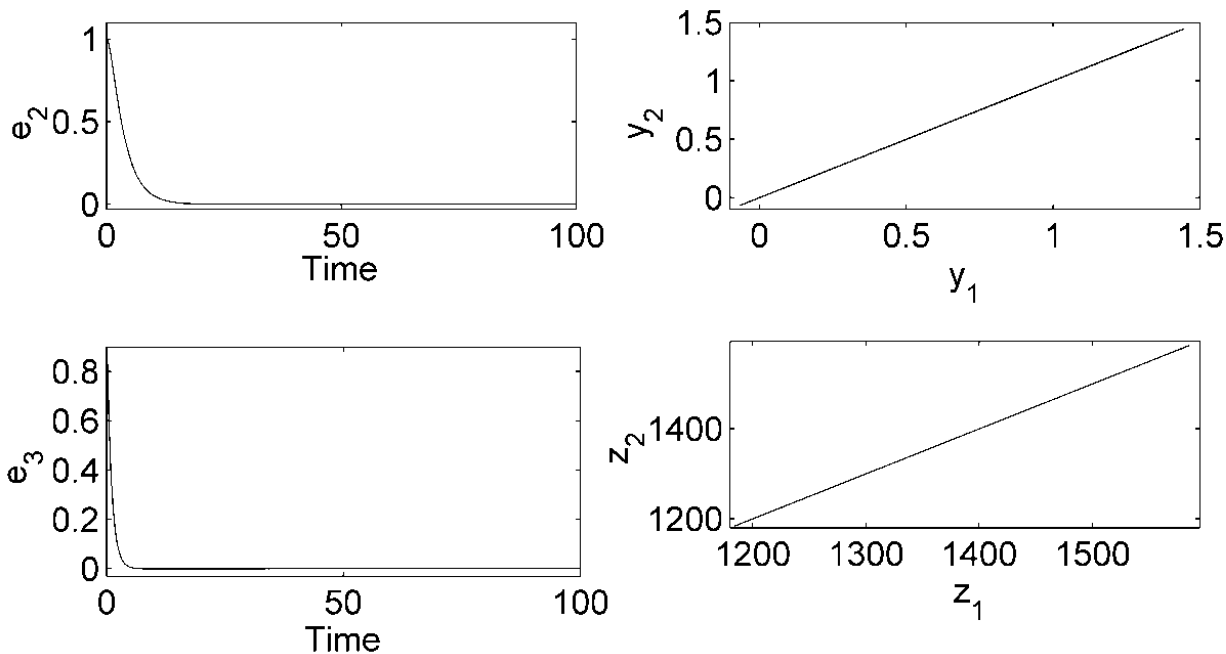

Fig. 6. Time evolutions of $e_{1}, e_{2}, e_{3}$ (a) and synchronization diagrams (b) showing synchronization for the values of parameters $q=1, i=1.15, \beta_{C}=0.707, \beta_{L}=3$.

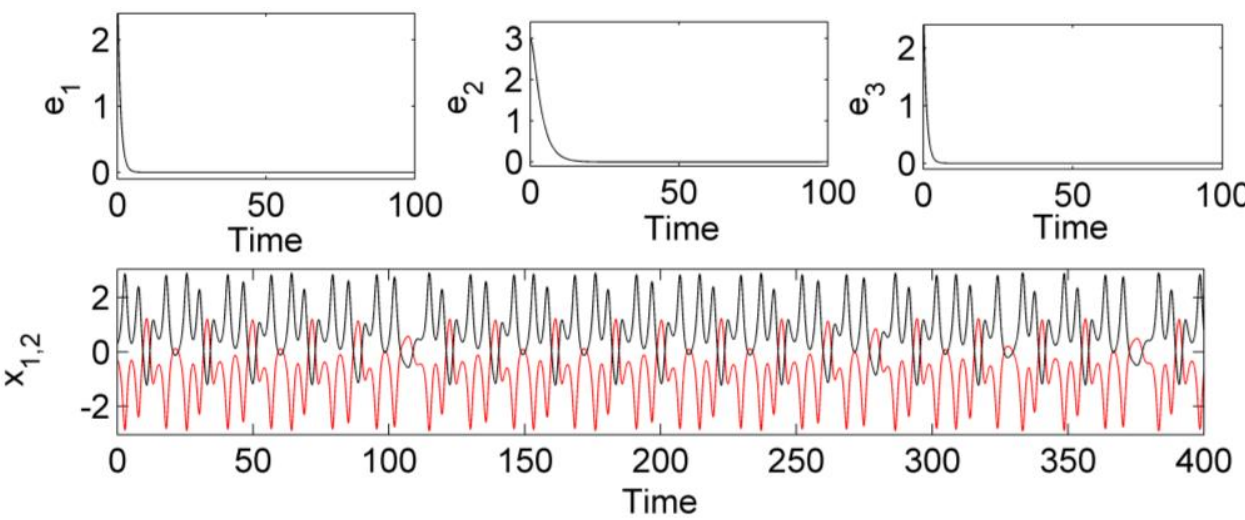

(a)

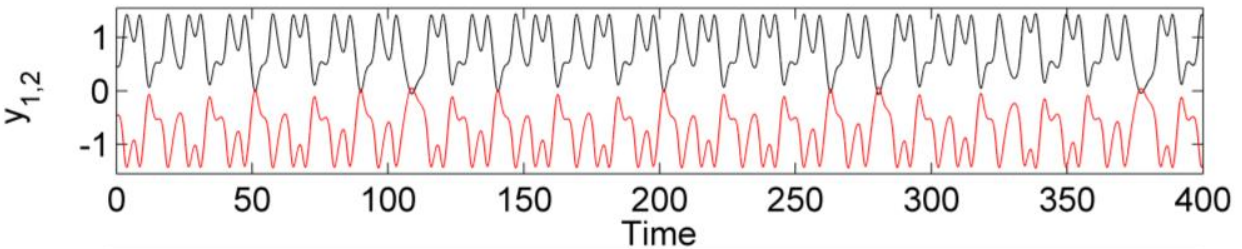

(b)

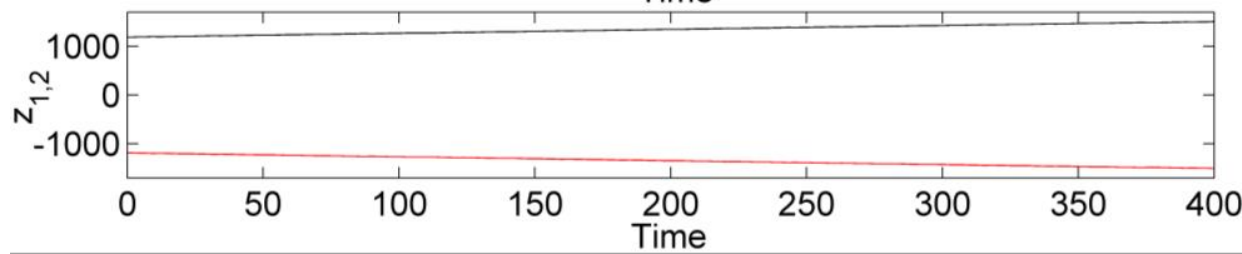

Fig. 7. Time evolutions of $e_{1}, e_{2}, e_{3}$ (a) and $x_{1,2}, y_{1,2}, z_{1,2}$ (b) showing antisynchronization between the drive and response for the values of parameters $q=-1, i=1.15, \beta_{C}=0.707, \beta_{L}=3$. 


\section{Conclusion}

The microcontroller implementation, chaos control, synchronization, and antisynchronization of the nonlinear resistive-capacitive-inductive shunted Josephson junction model was investigated in this paper. The existence of different shapes of hidden chaotic attractors was demonstrated by using numerical simulations and microcontroller implementation of nonlinear resistive-capacitive-inductive shunted Josephson junction model. Two single feedback controllers were designed to control chaos found in the nonlinear resistive-capacitive-inductive shunted Josephson junction model. Analytical calculations and numerical simulations are carried out to show the serviceableness of the two designed single controllers. The synchronization and antisynchronization of unidirectional coupled nonlinear resistive-capacitive-inductive shunted Josephson junction models were achieved by using the feedback control method.

\section{References}

[1] B. Soodchomshom, I. M. Tang, and R. Hoonsawat," Josephson effects in MgB2/ThinInsulator/MgB2 tunnel junction," Solid State Communications, vol. 149, no. 25-26 pp. 1012-1016, July 2009. https://doi.org/10.1016/j.ssc.2009.04.007

[2] B. D Josephson, "Non-linear conduction in superconductors," Doctoral Thesis, 1964. https://doi.org/10.17863/CAM.644

[3] J. Clarke, "A superconducting galvanometer employing Josephson tunnelling," Philosophical Magazine, vol. 13, pp. 115-127, August 2006. https://doi.org/10.1080/14786436608211991

[4] M. Suzuki, M. Hayashi, H. Ebisawa, "Nonlinear dynamics and resistive transition in intrinsic Josephson junctions.," J. Phys. Chem. Solid, vol. 69, pp. 3253-3256, December 2008. https://doi.org/10.1016/j.jpcs.2008.06.135

[5] G. V. Osipov, A. S. Pikovsky, and J. Kurths, "Phase Synchronization of Chaotic Rotators," Phys. Rev. Lett, vol. 88, pp. 054102, January 2002. https://journals.aps.org/prl/abstract/10.1103/PhysRevLett.88.054102

[6] V. K. Kornev and A. V. Arzumanov, "Josephson-junction oscillation spectral linewidth for some phase-locked multijunction systems,"J. Phys, vol. 8, pp. 279-282, June 1998. https://doi.org/10.1051/jp4:1998362

[7] A. Kanasugi, M. Morisue, H. Noguchi, M. Yamadaya, and H. Furukawa, "Oscillation modes in a josephson circuit and its application to digital systems," IEICE Transactions on Electronics, vol. E79-C, pp. 1206-1212, September 1996. https://search.ieice.org/bin/summary.php?id=e79c_9_1206

[8] E. N. Pozzo, D. Dominguez, "Fidelity and Quantum Chaos in the MesoscopicDevice for the Josephson Flux Qubit," Phys Rev. Lett, vol. 98, pp. 057006, February 2007. https://doi.org/10.1103/PhysRevLett.98.057006

[9] K. Inomata, S. Sato, K. Nakajima, A. Tanaka, H.B. Wang, M. Nagao, H. Hatano, S. Kawabata, "Macroscopic Quantum Tunneling in a d-Wave High-TC Bi2Sr2CaCu208+ $\delta$ Superconductor," Phys. Rev. Lett, vol. 95, pp. 107005, September 2005. https://doi.org/10.1103/PhysRevLett.95.107005

[10] M. Machida, T. Kano, S. Yamada, M. Okumora, T. Imamura, T. Koyama, "Quantum effects on capacitively coupled intrinsic Josephson junctions" J. Phys. Chem. Solids, vol. 69, pp. 3221-3224, December 2008. https://doi.org/10.1016/j.jpcs.2008.06.098

[11] Y. L. Feng and K. Shen, "Controlling Chaos in RLC Shunted Josephson Junction by delayed Linear Feedback," Chinese Physics B, vol. 17, pp. 111-116, 2008. https://doi.org/10.1088/1674$1056 / 17 / 1 / 020$

[12] S. P. Benz and C. A. Hamilton, "A pulse-driven programmable Josephson voltage standard," Applied physics letters, vol. 68, pp. 3171-3179, March 1996. https://doi.org/10.1063/1.115814

[13] K. K. Likharev and V. K. Semenov, "RSFQ logic/memory family: a new Josephson-junction technology for sub-terahertz-clock-frequency digital systems," IEEE Transactions on Applied Superconductivity, vol. 1, pp. 3-28, March 1991. https://DOI: 10.1109/77.80745 
[14] F. Piazza, L. A. Collins, A. Smerzi, "Current-phase relation of a Bose-Einstein condensate flowing through a weak link," Phy. Rev. A, vol. 81, pp. 033613, April 2010. https://doi.org/10.1103/PhysRevA.81.033613

[15] W. C. Stewart, "Current-Voltage characteristics of Josepfson Junctions," Appl. Phys. Lett, vol. 12, no. 277, 1968. https://doi.org/10.1063/1.1651991

[16] S. T. Kingni, G. FautsoKuiate, V. Kamdoum Tamba, V.-T. Pham, \& D. V. Hoang, "Self-excited and hidden attractors in autonomous Josephson jerk oscillator: Analysis and its application to text encryption," Journal of Computational and Nonlinear Dynamics, 2019. http://yetl.yabesh.ir/yetl1/handle/yetl/4259159

[17] F. C. Talla, R. Tchitnga, P. H. L. Fotso, R. Kengne, B. Nana, \& A. Fomethe, "Unexpected Behaviors in a Single Mesh Josephson Junction Based Self-Reproducing Autonomous System," International Journal of Bifurcation and Chaos, vol. 30, no. 07, 2050097, 2020. https://doi.org/10.1142/s0218127420500972

[18] S. T. Kingni, G. F. Kuiate, R. Kengne, R. Tchitnga and Paul Woafo, "Analysis of a no equilibrium linear resistive-capacitive-inductance shunted junction model, dynamics, synchronization and application to Digital cryptography in its fractional-order form," Complexity, vol. 2017, Article ID 4107358, 2017. https://doi.org/10.1155/2017/4107358

[19] F. Salam and S. Sastry, "Dynamics of the forced Josephson junction circuit: the regions of chaos," IEEE Transactions on Circuits and Systems, vol. 32, no. 8, pp. 784-796, August 1985. https://doi.org/10.1109/TCS.1985.1085790

[20] C. B. Whan, C. J. Lobb, and M. G. Forrester, "Effect of inductance in externally shunted Josephson tunnel junctions," Journal of Applied Physics, vol. 77, pp. 382, September 1994. https://doi.org/10.1063/1.359334

[21] A. B. cawthorne, C. B. Whan, and C. J. lobb, "complex dynamics of resistively and inductively shunted josephson junctions," Journal of Applied Physics, vol. 84, pp. 1126-1132, April 1998. https://doi.org/10.1063/1.368113

[22] B. Ramakrishnan, A. Durdu, K. Rajagopal, \& A. Akgul, "Infinite attractors in a chaotic circuit with exponential memristor and Josephson junction resonator," AEU - International Journal of Electronics and Communications, vol. 123, 153319, 2020. https://doi.org/10.1016/j.aeue.2020.153319

[23] S. Vaidyanathan, S. Takougang Kingni, A. Sambas, M. Afendee Mohamed, M. Mamat, "A new chaotic jerk system with three nonlinearities and synchronization via adaptive backstepping control," International Journal of Engineering and Technology, vol. 7, no. 3, pp. 1936-1943, 2018. https://doi.org/10.14419/ijet.v7i3.15378

[24] S. T. Kingni, K. Rajagopal, V. K. Tamba, C. Ainamon, \& J. B. C. Orou, "Analysis and FPGA implementation of an autonomous Josephson junction snap oscillator," The European Physical Journal B, vol. 92, Article number 227, 2019. https://doi.org/10.1140/epjb/e2019-100304-X

[25] U. E. Vincent, A. Ucar, J. Alaoye, S. O. Kareem, "control and synchronization of chaos in rclshunted josephson junction using backstepping design," Physica C: Superconductivity, vol. 468, no. 5, pp. 374-382, 2008. https://doi.org/10.1016/j.physc.2007.11.012

[26] A. M. Harb, and B. A. Harb, "Controlling chaos in Josephson-junction using nonlinear backstepping controller," IEEE transactions on applied superconductivity, vol. 16, no. 4, pp. 19881998, 2006. https://doi.org/10.1109/TASC.2006.881811

[27] A. N. Njah, K. S. Ojo, G. A. Adebayo, A. O. Obawole, "Generalized control and synchronization of chaos in RCL-shunted josephson junction using backstepping design," Physica C: Superconductivity, vol. 470, pp. 558-564, 2010. https://doi.org/10.1016/j.physc.2010.05.009

[28] D. Y. Chen, W. L. Zhao, X. Y. Ma and R. F. Zhang, "control and synchronization of chaos in rclshunted josephson junction with noise disturbance using only one controller term," Abstract and Applied Analysis, vol. 2012, article ID 378457, 2012. https://doi.org/10.1155/2012/378457

[29] Y. L. Feng, X. H. Zhang, Z. G. Jian and K. Shen, "Controlling chaos in rcl-shunted Josephson junction by delayed linear feedback," International Journal of Modern Physics B, vol. 23, no. 18, pp. 3803-3809, 2009. https://doi.org/10.1142/S0217979209052698

[30] N. N. Wang, H. Zhao, X. R. Wang and H. T. Liang, "The chaotic control with only one controller term of josephson junction system," Advanced Materials Research, vol. 462, pp. 866-872, 2012. https://doi.org/10.4028/www.scientific.net/AMR.462.866 